\title{
Prognostic significance of CXCL5 expression in cancer patients: a meta-analysis
}

\author{
Binwu Hu ${ }^{1 \dagger}$, Huiqian Fan ${ }^{2+}$, Xiao Lv ${ }^{1}$, Songfeng Chen $^{3}$ and Zengwu Shao ${ }^{1 *} \mathbb{C}$
}

\begin{abstract}
Background: CXCL5 is a member of the CXC-type chemokine family, which has been found to play important roles in tumorigenesis and cancer progression. Recent studies have demonstrated that CXCL5 could serve as a potential prognostic biomarker for cancer patients. However, the prognostic value of CXCL5 is still controversial.

Methods: We systematically searched PubMed, Embase and Web of Science to obtain all relevant articles investigating the prognostic significance of CXCL5 expression in cancer patients. Hazards ratios (HR) with corresponding 95\% confidence intervals $(\mathrm{Cl})$ were pooled to estimate the association between CXCL5 expression levels with survival of cancer patients.

Results: A total of 15 eligible studies including 19 cohorts and 5070 patients were enrolled in the current metaanalysis. Our results demonstrated that elevated expression level of CXCL5 was significantly associated with poor overall survival (OS) (pooled HR 1.70; 95\% Cl 1.36-2.12), progression-free survival (pooled HR 1.65; 95\% Cl 1.09-2.49) and recurrence-free survival (pooled HR 1.49; $95 \% \mathrm{Cl} 1.15-1.93$ ) in cancer patients. However, high or low expression of CXCL5 made no difference in predicting the disease-free survival (pooled HR 0.63; 95\% Cl 0.11-3.49) of cancer patients. Furthermore, we found that high CXCL5 expression was associated with reduced OS in intrahepatic cholangiocarcinoma (HR 1.91; 95\% Cl 1.31-2.78) and hepatocellular carcinoma (HR 1.87; 95\% Cl 1.55-2.27). However, there was no significant association between expression level of CXCL5 with the OS in lung cancer ( $\mathrm{HR} 1.25 ; 95 \% \mathrm{Cl}$ 0.79-1.99) and colorectal cancer ( $\mathrm{HR} 1.16 ; 95 \% \mathrm{Cl} 0.32-4.22, \mathrm{p}=0.826$ ) in current meta-analysis.
\end{abstract}

Conclusions: In conclusion, our meta-analysis suggested that elevated CXCL5 expression might be an adverse prognostic marker for cancer patients, which could help the clinical decision making process.

Keywords: Chemokine, CXCL5, Cancer, Prognosis, Meta-analysis

\section{Background}

Despite great improvements in early detection, surgical techniques, chemotherapy, radiotherapy, biological treatment and multidisciplinary treatment in recent years, cancer is still a major public health problem globally, which is associated with high morbidity, mortality and economic burden [1]. It is estimated that $1,735,350$ new cancer cases and 609,640 cancer deaths are projected to occur in the United States in 2018 [2]. Given the poor prognosis of cancer patients, numerous investigators

\footnotetext{
*Correspondence: szwpro@163.com

${ }^{+}$Binwu Hu and Huiqian Fan contributed equally to this work

1 Department of Orthopaedics, Union Hospital, Tongji Medical College, Huazhong University of Science and Technology, Wuhan 430022, China Full list of author information is available at the end of the article
}

have focused on searching for biomarkers that could predict prognosis of cancer. However, sensitivity and specificity of most cancer biomarkers widely used now are not yet satisfactory [3]. Therefore, it is desperately needed to identify novel applicable prognostic biomarkers, not only improving poor prognosis but also providing novel therapeutic targets.

Chemokines are chemotactic cytokines that could regulate the migration of immune cells into damaged or diseased organs in response to pro-inflammatory stimuli [4]. According to cysteine residues in the NH2-terminal part of the protein, chemokines can be classified into four highly conserved groups, namely $\mathrm{C}, \mathrm{CC}, \mathrm{CXC}$, and CX3C [5]. Chemokines and their receptors could bring about the transcription of target genes involved in cell invasion, 
motility, survival and interactions with the extracellular matrix, which can induce migration, chemotaxis and rearrangement of the cytoskeleton in the target cell, and therefore promote multiple physiological functions of cells, including cell growth, development, differentiation and apoptosis [6-9]. Over the past few years, accumulating evidence has revealed that chemokines play pivotal roles in progression of tumor [10]. Chemokines produced by tumor and stromal cells can induce the expression and distribution of tumor-associated leukocytes, trigger angiogenesis, contribute to the growth and metastasis of malignant cells and generate fiber keratinocytes [6, $11,12]$. In addition, chemokines and their receptors are critical mediators of inflammation microenvironment of cancer, which has been proposed to represent the seventh hallmark of cancer [13, 14]. Given the important roles of chemokines in cancer, abnormal expression of chemokines has been detected in many tumors, and several chemokines have been proven to be associated with poor prognosis of cancer patients [15-17].

CXCL5, also known as epithelial-derived neutrophilactivating peptide 78 (ENA78), is originally discovered as a potent chemoattractant and activator of neutrophil function. Through binding to its receptor CXCR2, CXCL5 could induce the chemotaxis of neutrophils, promote angiogenesis, and remodel connective tissue [18]. Accumulating evidence suggests that CXCL5 may participate in cancer-related inflammation, which is involved in many aspects of malignancy in cancer biology [19]. Furthermore, abnormal expression of CXCL5 has been identified in many tumors. CXCL5 is overexpressed in gastric cancer, prostate cancer, endometrial cancer, squamous cell cancer, hepatocellular carcinoma and pancreatic cancer, and increased expression of CXCL5 is associated with advanced tumor stages, local invasion, neutrophil infiltration and metastatic potential [20-25]. Recent studies have revealed that CXCL5 could serve as a potential prognostic biomarker for patients with cancer $[5,19$, $26,27]$. However, its prognostic value is still controversial owing to the fact that most studies reported so far are limited in discrete outcome and sample size. Therefore, we performed the current quantitative meta-analysis to elucidate the prognostic significance of CXCL5 expression in cancer patients.

\section{Materials and methods Study strategy}

The present review was performed in accordance with the standard guidelines for meta-analysis and systematic reviews of tumor marker prognostic studies [28, 29]. The database Web of Science, PubMed and Embase were independently searched by two researchers (Binwu $\mathrm{Hu}$ and Huiqian Fan) to obtain all relevant articles about the prognostic value of CXCL5 in patients with any tumor. The literature search ended on March 1, 2018. The search strategy used both MeSH terminology and freetext words to increase the sensitivity of the search. The search strategy was: "CXCL5 or CXC chemokine ligand 5 or ENA78 or epithelial cell derived neutrophil attractant 78 " AND "cancer or tumor or carcinoma or neoplasm or malignancy" AND "prognostic or prognosis or survival or outcome". We also screened the references of retrieved relevant articles to identify potentially eligible literatures. Conflicts were solved through group discussion.

\section{Inclusion and exclusion criteria}

Studies included in this analysis had to meet the following inclusion criteria: (1) patients were pathologically diagnosed with any type of human cancer. (2) CXCL5 expression levels were determined in human tissues or plasma samples. (3) Patients were divided into two groups according to the expression levels of CXCL5, the relationship between CXCL5 expression levels with survival outcome was investigated. (4) Sufficient published data or the survival curve were provided to calculate hazard ratios (HR) for survival rates and their 95\% confidence intervals $(\mathrm{CI})$. Exclusion criteria were as follow: studies using non-human samples, studies without usable or sufficient data, laboratory articles, reviews, letters, case reports, non-English or unpublished articles and conference abstracts. All eligible studies were carefully screened by two researchers (Binwu $\mathrm{Hu}$ and Huiqian Fan), and discrepancies were resolved by discussing with a third researcher (Xiao Lv).

\section{Data extraction}

Two investigators (Binwu Hu and Huiqian Fan) extracted relevant data independently and reached a consensus on all items. For all eligible studies, the following information of each article was collected: author, year of publication, tumor type, samples detected, expression associated with poor prognosis, Newcastle-Ottawa Scale (NOS) score, method of obtaining HRs, characteristics of the study population (including country of the population enrolled, number of patients (high/low), follow up (month)), endpoints, assay method, cut-off value and survival analysis. For endpoints, overall survival (OS), disease-free survival (DFS), progression-free survival (PFS) and recurrence-free survival (RFS) were all regarded as endpoints. We employed HR which was extracted following a methodology suggested previously to evaluate the influence of CXCL5 expression on prognosis of patients [30]. If possible, we also asked for original data directly from the authors of the relevant studies. 


\section{Quality assessment}

Quality of all included studies was assessed independently by two researchers (Binwu $\mathrm{Hu}$ and Huiqian Fan) using the validated Newcastle-Ottawa Scale, and disagreements were resolved through discussion with another researcher (Songfeng Chen). This scale uses a star system to evaluate a study in three domains: selection of participants, comparability of study groups, and the ascertainment of outcomes of interest. We considered studies with scores more than 6 as high-quality studies, and those with scores no more than 6 as lowquality studies.

\section{Statistical analysis}

Statistical analysis was performed using Stata Software 14.0 (Stata, College Station, TX). Pooled HRs (high/low) and their associated $95 \%$ CIs were used to analyze the prognostic role of CXCL5 expression in various cancers. The heterogeneity among studies was evaluated using Cochran's $Q$ and $I^{2}$ statistics. A p value less than 0.10 or an $\mathrm{I}^{2}$ value larger than $50 \%$ were considered statistically significant. The fixed-effect model was used for analysis without significant heterogeneity between studies $\left(\mathrm{p}>0.10, \mathrm{I}^{2}<50 \%\right)$. Otherwise, the random-effect model was chosen. To explore the source of heterogeneity, subgroup analysis and meta-regression were preformed through classifying the included studies into subgroups according to similar features. We also conducted sensitivity analysis to test the effect of each study on the overall pooled results. The publication bias was evaluated by using both Begg's test and Egger's test. A p value less than 0.05 was considered statistically significant.

\section{Results}

\section{Characteristics of studies}

According to our search strategy, the initial search algorithm retrieved a total of 554 studies. The following studies were excluded: duplicates $(n=196)$, review $(n=14)$, patent $(n=9)$, meeting abstract $(n=64)$, studies describing non-cancer topics $(\mathrm{n}=27)$, studies describing non-CXCL5 topics $(n=126)$, studies belonging to basic research $(n=75)$, studies lacking relevant data $(n=26)$ and non-English articles $(\mathrm{n}=2)$. Eventually, 15 studies meeting the inclusion criteria were included in this metaanalysis. The screening process and results are shown in Fig. 1.

The main characteristics of the included studies are shown in Table 1. A total of 15 studies including 19 cohorts were included in the current metaanalysis. Among these studies, a total of 5070 patients were included, with a minimum sample size of 27 and a maximum sample size of 2437 patients. The accrual

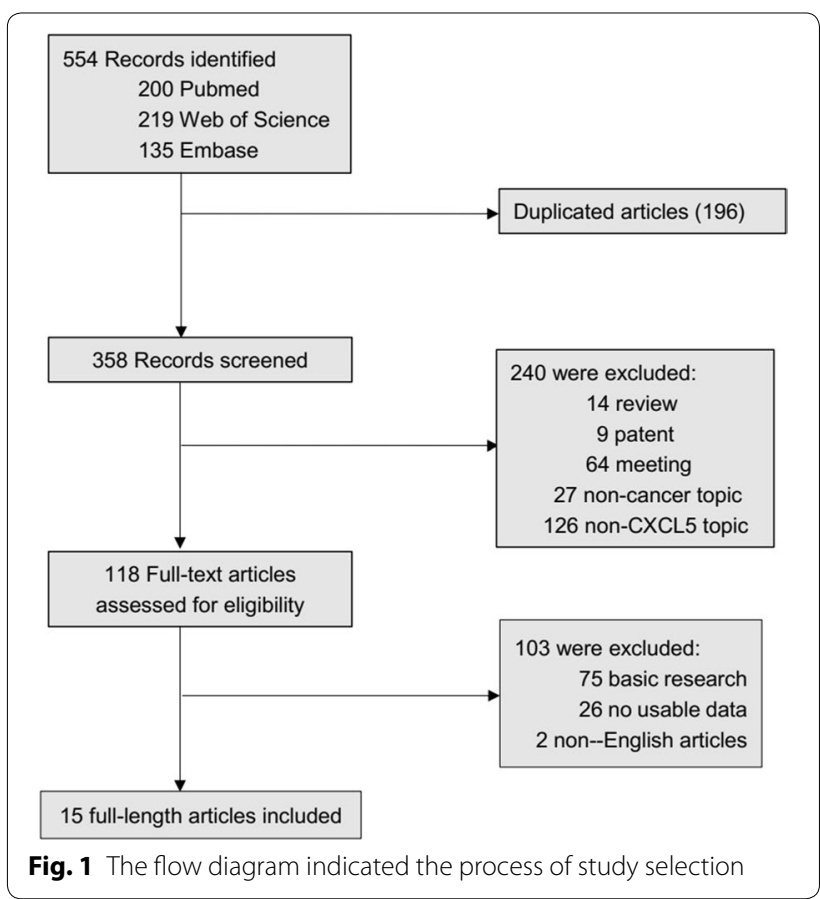

period of these studies ranged from 2007 to 2018. The follow-up time ranged from 23 months to 180 months. Ten different types of cancer were involved in the enrolled studies including intrahepatic cholangiocarcinoma $(n=2)[27,31]$, lung cancer $(n=3)[26,32,33]$, colorectal cancer $(n=3)$ [34-36], biliary tract cancer $(n=1)$ [10], breast cancer $(n=1)$ [5], bladder cancer $(n=1)$ [19], glioma $(n=1)$ [18], pancreatic cancer $(n=1)$ [25], hepatocellular carcinoma $(n=1)$ [24] and nasopharyngeal carcinoma $(n=1)$ [37]. Among these studies, OS $(n=14)$, DFS $(n=3)$, PFS $(n=3)$ and RFS $(n=3)$ were estimated as survival outcome. The CXCL5 expression levels in these studies were mostly measured by using immunohistochemistry (IHC) technique, while real time PCR (RT-PCR) and enzyme-linked immunosorbent assay (ELISA) were also applied. Because the cut-off definitions were various, the cut-off values were different in these studies.

\section{Association between CXCL5 expression levels with OS of cancer patients}

Fourteen studies including seventeen cohorts reported the relationship between abnormal expression levels of CXCL5 with OS in a total of 4952 cancer patients. We used random-effect model to calculate the pooled HR. The pooled HR for OS was 1.70 (95\% CI 1.36-2.12, $\mathrm{p}<0.001)$, which suggested that elevated expression level of CXCL5 was significantly associated with poor OS in cancer patients (Fig. 2). Given that significant 


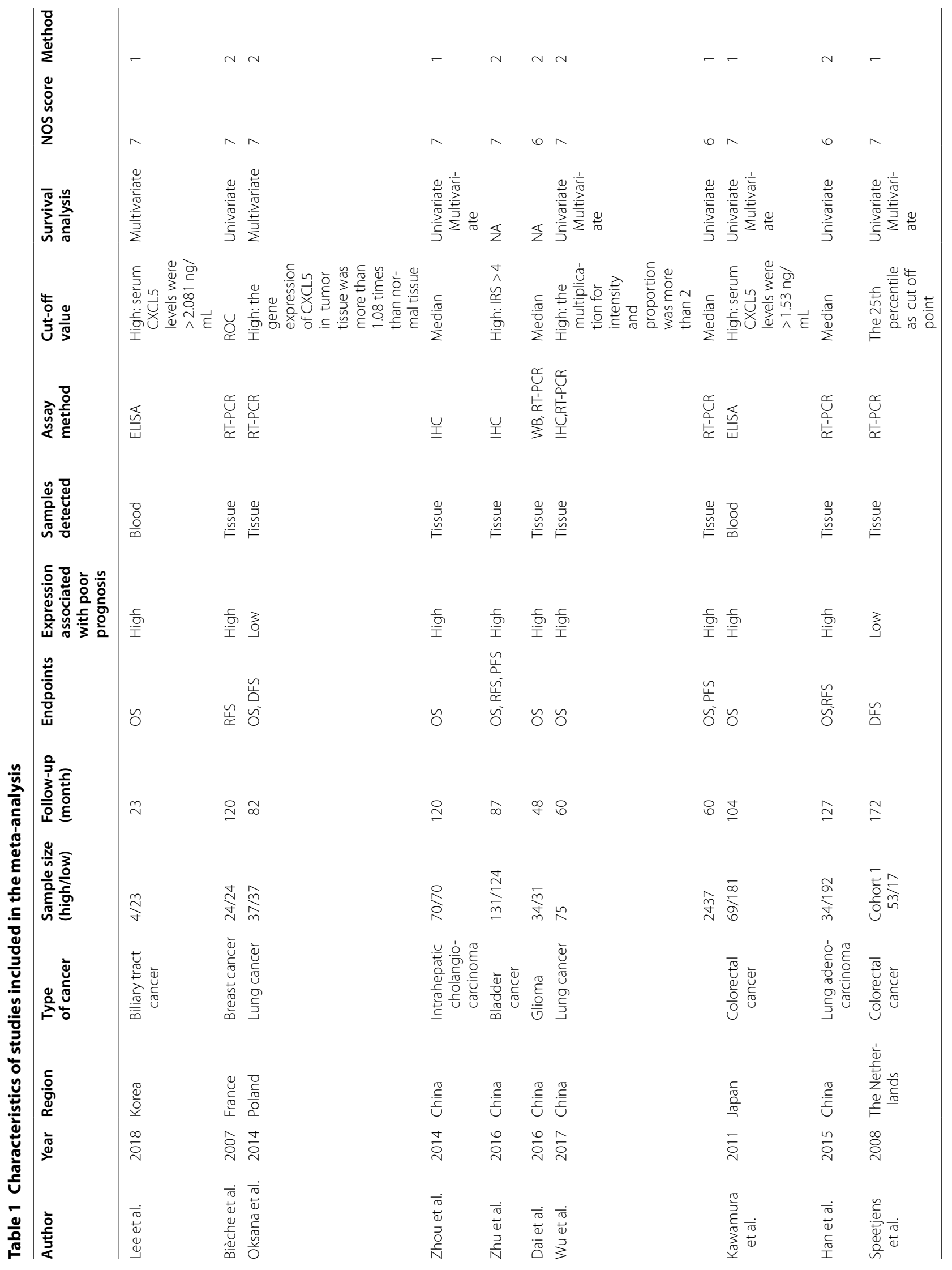




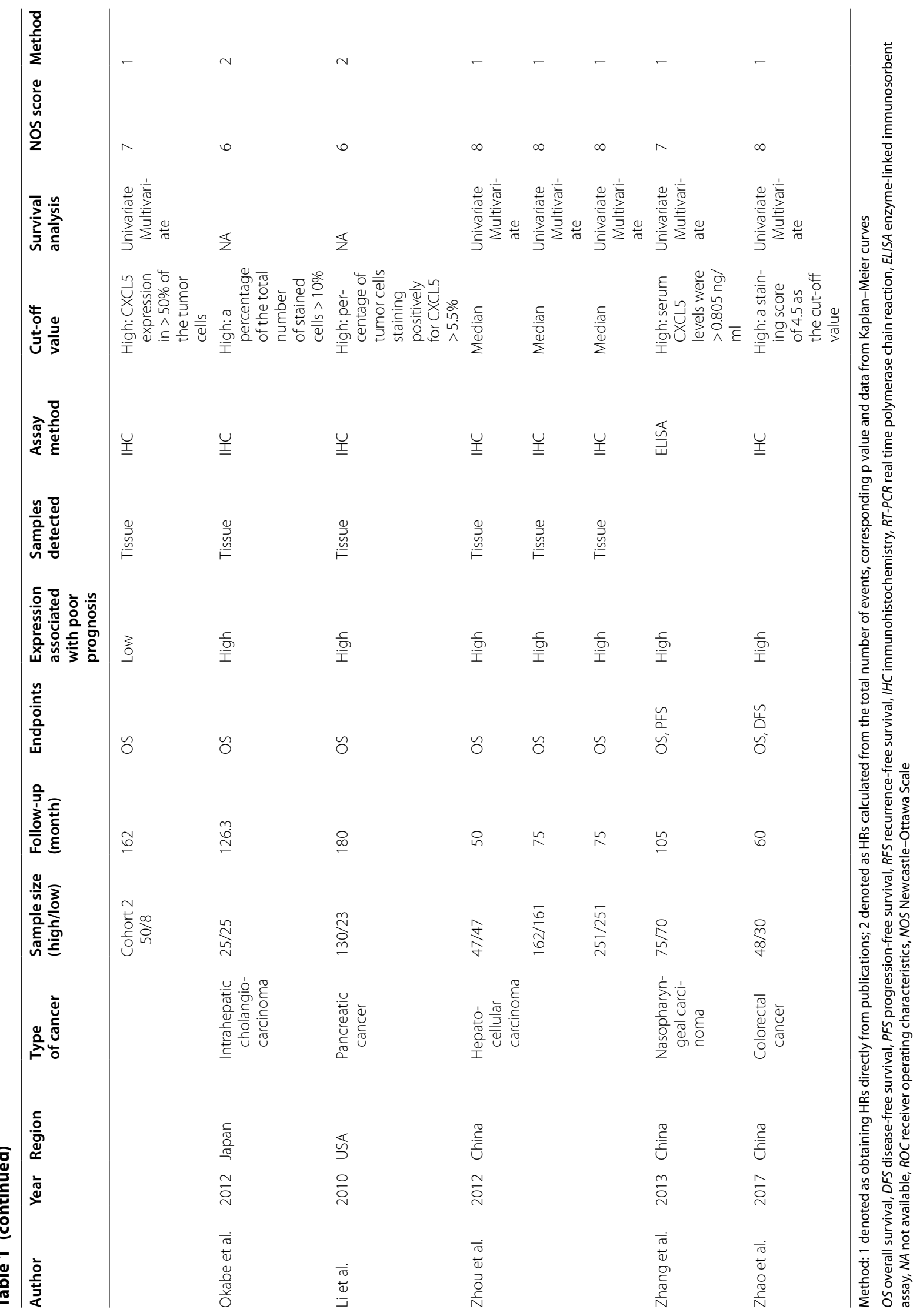




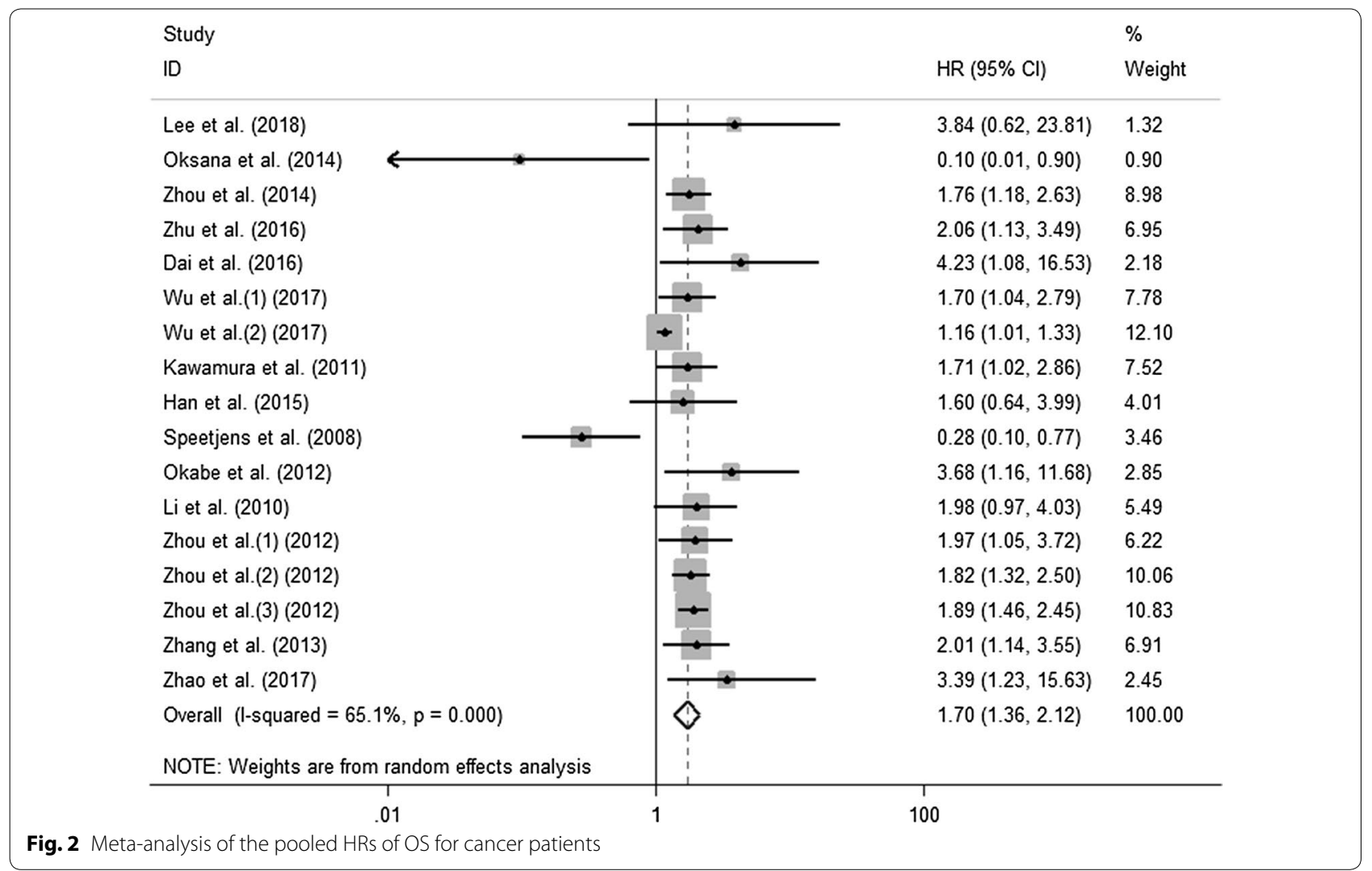

heterogeneity existed among studies $\left(\mathrm{I}^{2}=65.1 \%\right.$; $\mathrm{p}<0.001$ ), we further conducted subgroup analysis by factors of sample size (fewer than 100 or more than 100), type of cancer (digestive system or non-digestive system carcinoma), follow-up time (fewer than 100 or more than 100 months), samples detected (blood or tissue), paper quality (NOS scores $\geq 7$ or $<7$ ) and source of HR (directly or indirectly) to explore the source of heterogeneity (Fig. 3a-f). The results of subgroup analysis illustrated that the association between increased expression level of CXCL5 with poor OS of cancer patients was still significant in all factors above except for the subgroup of studies with fewer than 100 patients (HR 1.60, 95\% CI 0.81-3.17, $\mathrm{p}=0.175$ ) (Table 2). To further explore the sources of heterogeneity, we performed meta-regression by the covariates including above factors. However, metaregression didn't reveal $\mathrm{p}$ values less than 0.05 in above covariates, which indicated that all above factors were not the sources of heterogeneity (Table 2). Furthermore, using Cox multivariate analysis in eight studies including ten cohorts, we found that elevated CXCL5 expression levels was an independent prognostic factor for OS in cancer patients (HR 1.65, 95\% CI 1.24-2.20, $\mathrm{p}=0.001$ ).

\section{Association between CXCL5 expression levels with OS of certain types of cancer}

We further evaluated the prognostic value of CXCL5 in certain types of cancer. Through systematic analysis, our results demonstrated that high CXCL5 expression was associated with reduced OS in intrahepatic cholangiocarcinoma (HR 1.91; 95\% CI 1.31-2.78, p=0.001) (Fig. 4a) and hepatocellular carcinoma (HR 1.87; 95\% CI 1.552.27, $\mathrm{p}<0.001$ ) (Fig. 4b). However, there was no significant association between expression level of CXCL5 with $\mathrm{OS}$ of cancer patients in lung cancer (HR 1.25; 95\% CI $0.79-1.99, \mathrm{p}=0.335$ ) (Fig. 4c) and colorectal cancer (HR 1.16; 95\% CI 0.32-4.22, $\mathrm{p}=0.826$ ) (Fig. 4d).

\section{Association between CXCL5 expression levels with DFS, PFS and RFS of cancer patients}

There were three studies respectively evaluating the relationship between CXCL5 expression levels with DFS, PFS and RFS. Through systematic analysis, our results revealed that higher expression level of CXCL5 was significantly associated with shorter PFS (HR 1.65; 95\% CI 1.09-2.49, $\mathrm{p}=0.018$ ) (Fig. 5a) and RFS (HR 1.49; 95\% CI $1.15-1.93, \mathrm{p}=0.003$ ) (Fig. $5 \mathrm{~b}$ ). However, high or low 

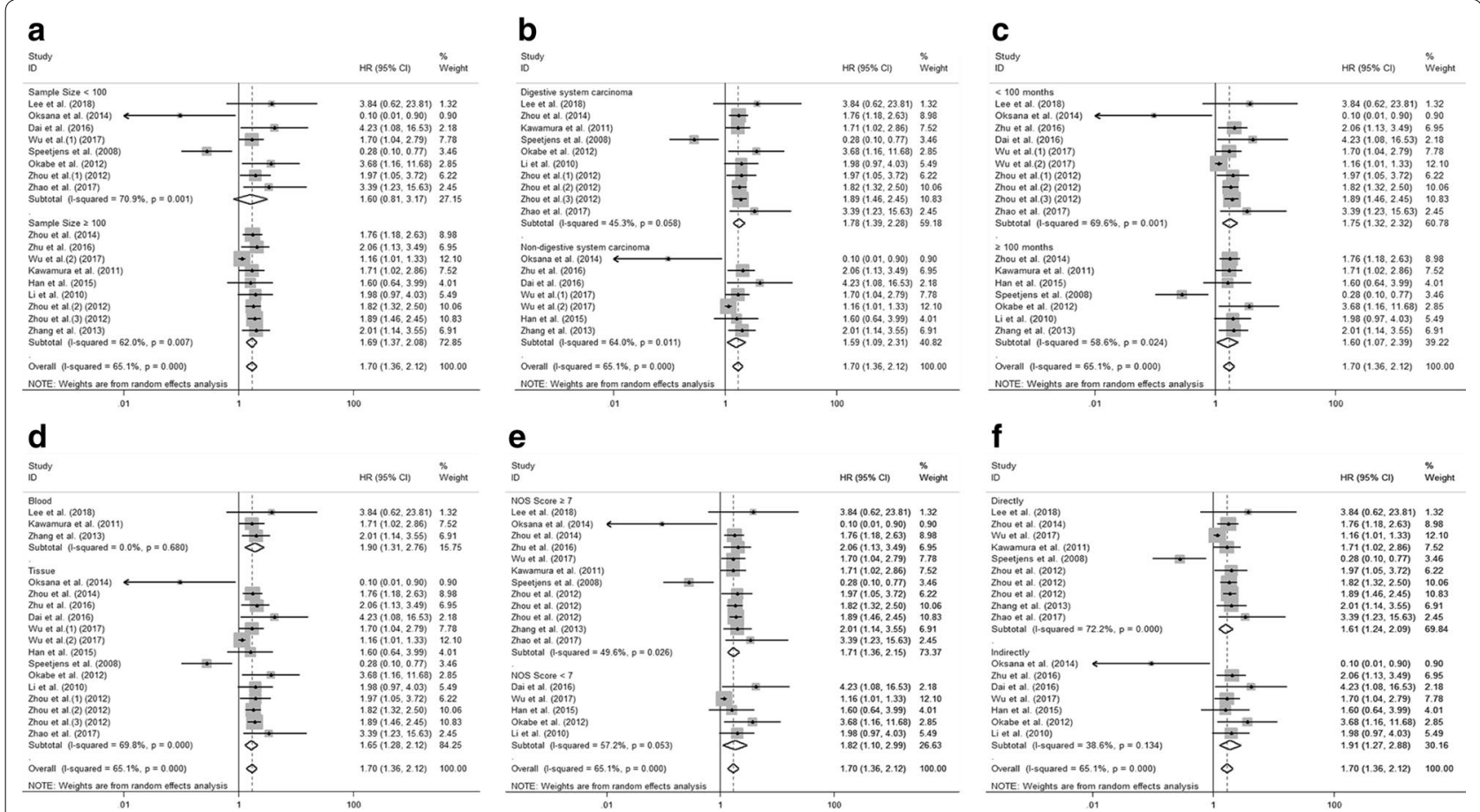

Fig. 3 Results of subgroup analysis of pooled HRs of OS for cancer patients. a Subgroup analysis stratified by sample size. b Subgroup analysis stratified by type of cancer. $\mathbf{c}$ Subgroup analysis stratified by follow-up time. $\mathbf{d}$ Subgroup analysis stratified by sample detected. e Subgroup analysis stratified by NOS score. $\mathbf{f}$ Subgroup analysis stratified by source of HR

Table 2 Subgroup analysis of pooled HRs for OS in cancer patients with abnormal expression level of CXCL5

\begin{tabular}{|c|c|c|c|c|c|}
\hline \multirow[t]{2}{*}{ Subgroup analysis } & \multirow[t]{2}{*}{ No. of cohorts } & \multirow{2}{*}{$\begin{array}{l}\text { Pooled HRs } \\
\text { Random }\end{array}$} & \multirow[t]{2}{*}{ Meta regression ( $p$ value) } & \multicolumn{2}{|c|}{ Heterogeneity } \\
\hline & & & & $I^{2}(\%)$ & $p$ value \\
\hline Sample size & & & 0.602 & & \\
\hline$<100$ & 8 & $1.60[0.81-3.17]$ & - & 70.9 & 0.001 \\
\hline$\geq 100$ & 9 & $1.69[1.37-2.08]$ & - & 62.0 & 0.007 \\
\hline Type of cancer & & & 0.197 & & \\
\hline Digestive system carcinoma & 10 & $1.78[1.39-2.28]$ & - & 45.3 & 0.058 \\
\hline Non-digestive system carcinoma & 7 & $1.59[1.09-2.31]$ & - & 64.0 & 0.011 \\
\hline Follow-up time & & & 0.204 & & \\
\hline$<100$ & 10 & $1.75[1.32-2.32]$ & - & 69.6 & 0.001 \\
\hline$\geq 100$ & 7 & $1.60[1.07-2.39]$ & - & 58.6 & 0.024 \\
\hline Samples detected & & & 0.186 & & \\
\hline Blood & 3 & $1.90[1.31-2.76]$ & - & 0.0 & 0.680 \\
\hline Tissue & 14 & $1.65[1.28-2.12]$ & - & 69.8 & 0.000 \\
\hline NOS score & & & 0.526 & & \\
\hline$\geq 7$ & 12 & $1.71[1.36-2.15]$ & - & 49.6 & 0.026 \\
\hline$<7$ & 5 & 1.82 [1.10-2.99] & - & 57.2 & 0.053 \\
\hline Source of HR & & & 0.209 & & \\
\hline Directly & 10 & 1.61 [1.24-2.09] & - & 72.2 & 0.000 \\
\hline Indirectly & 7 & $1.91[1.27-2.88]$ & - & 38.6 & 0.134 \\
\hline
\end{tabular}



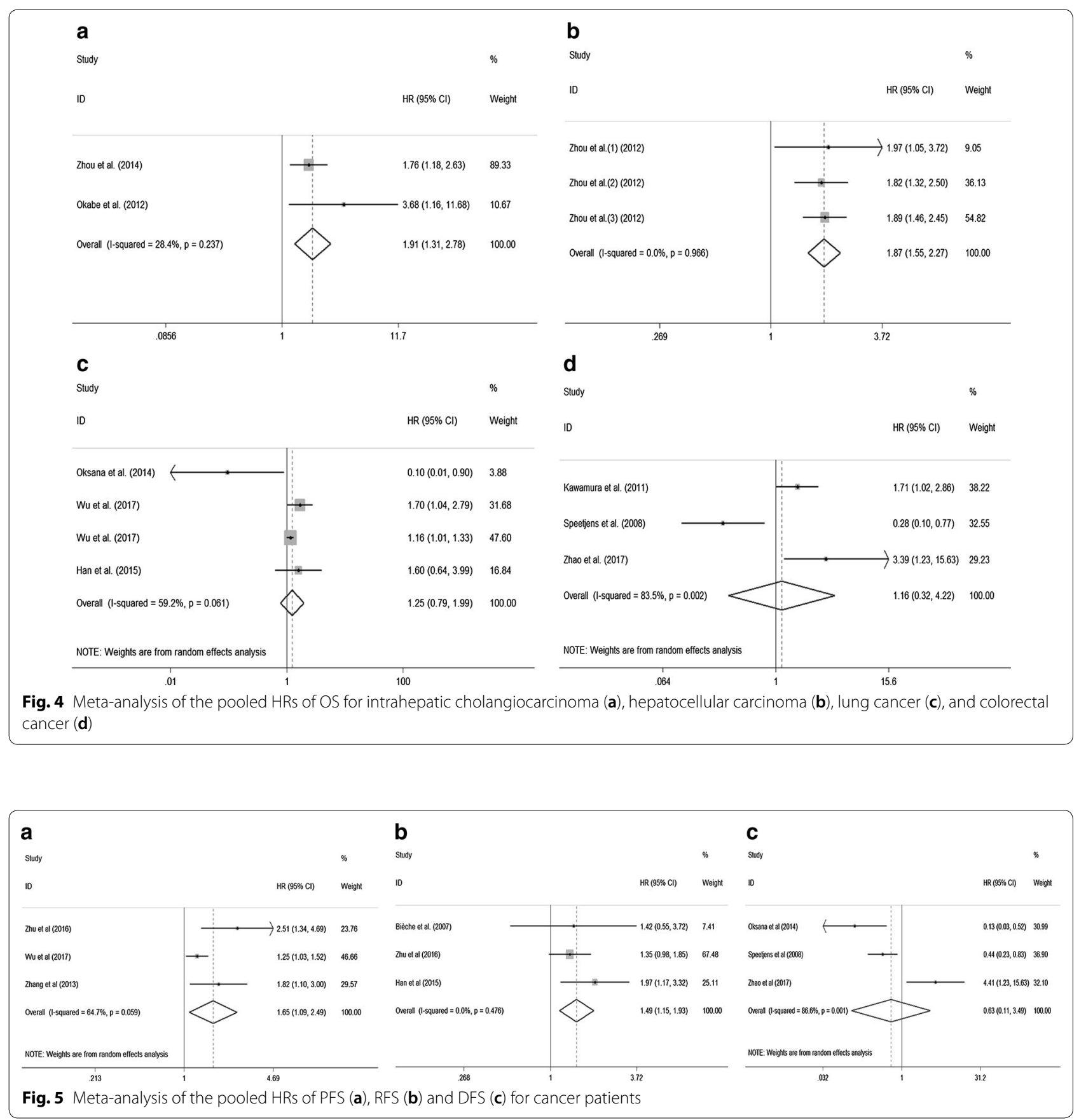

expression of CXCL5 made no difference in predicting the DFS (HR 0.63; 95\% CI 0.11-3.49, $\mathrm{p}=0.595$ ) (Fig. 5c). In addition, due to the limited number of included studies, we did not perform the subgroup analysis.

\section{Sensitivity analysis and publication bias}

We performed sensitivity analysis to examine the effects of individual study on the overall results. For OS, the sensitivity analysis identified that results from Wu et al.
(2) and Speetjens et al. affected results greatly, which indicated that these two studies were possible to be the main source of heterogeneity. However, the list of pooled HRs and $95 \%$ CIs after excluding single study one by one indicated robustness of our results, in which all pooled HRs and 95\% CIs were above the null hypothesis of 1 (Fig. 6a). For DFS (Fig. 6b) and PFS (Fig. 6c), the sensitivity analysis revealed that all included studies affected results greatly. For RFS, only the results from Bièche et al. 

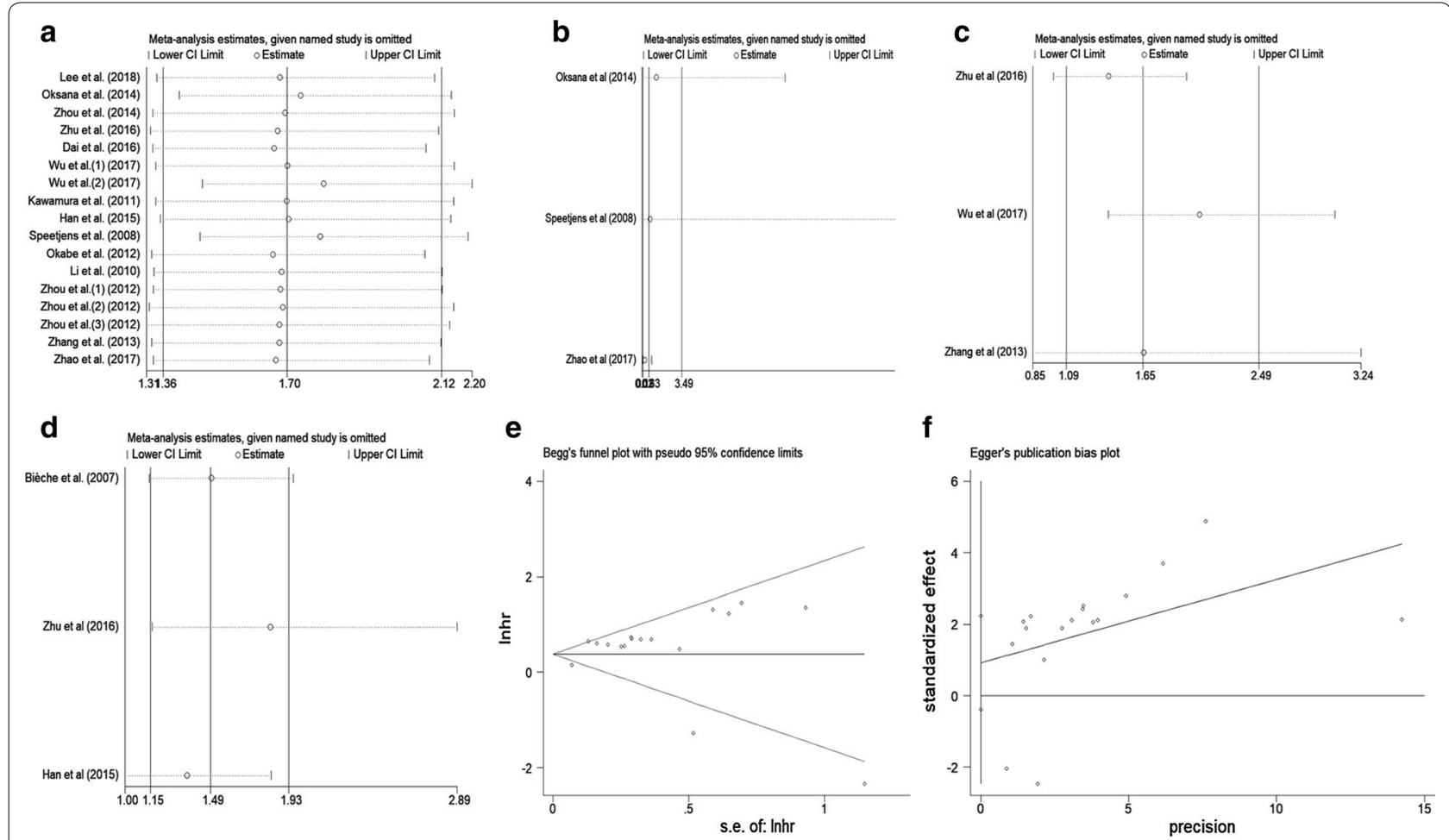

Fig. 6 Sensitivity analysis plot of pooled HRs of OS (a), DFS (b), PFS (c) and RFS (d) for cancer patients with abnormally expressed CXCL5. Begg's test (e) and Egger's test (f) for publication bias

did not influence the results greatly (Fig. 6d). The sensitivity analysis results demonstrated that our results for DFS, PFS and RFS were not that stable, which might be because of the limited number of studies included in each analysis. Therefore, more relevant studies are warranted to investigate the effects of CXCL5 on DFS, PFS and RFS in human cancer.

Begg's test and Egger's linear regression test were conducted to evaluate publication bias. For OS, Begg's test $(\mathrm{p}=0.773)$ (Fig. 6e) and Egger's test $(\mathrm{p}=0.157)$ (Fig. 6f) showed no significant publication bias across studies. For DFS, PFS and RFS, because of the limited number of studies (below 10) included in each analysis, publication bias was not assessed.

\section{Discussion}

CXCL5 is originally discovered as a potent chemoattractant and activator of neutrophil function [33]. Through interacting with CXCR2 receptor, it could function both as a chemoattractant and as an angiogenic factor $[35,38$, 39]. Recently, CXCL 5 has been shown to be able to promote the proliferation, migration and invasion of various tumor cells and play pivotal roles in the pathogenesis and progression of cancer [27,37]. It was reported that CXCL5 protein was higher in various lung cancer tissues, which was positively associated with tumor stage, lymph node metastasis, and worse survival [26] [19, 40]. Zhou et al. also reported that CXCL5 was overexpressed in intrahepatic cholangiocarcinoma cell lines and tumor samples, which could promote intrahepatic cholangiocarcinoma growth and metastasis by recruiting intratumoral neutrophils [27]. Furthermore, CXCL5 could directly induce endothelial cell proliferation and invasion in vitro and promote tumor angiogenesis in nonsmall cell lung carcinoma and pancreatic cancer [41-43]. Considering the important functions of CXCL5 in cancer, studies have demonstrated that CXCL5 could serve as a potential prognostic biomarker for cancer patients. However, the prognostic value of CXCL5 is still controversial. Because even in the same type of tumor, there are almost opposite conclusions about the prognostic value of CXCL5 [34-36].

Here we performed the current comprehensive metaanalysis to systematically explore the prognostic value of abnormally expressed CXCL5 in cancer patients. We examined 15 independent studies including 19 cohorts and 5070 patients. Through systematic analysis, our results demonstrated that high expression level of CXCL5 was significantly associated with poor OS in cancer patients. Due to the significant heterogeneity across these studies, we performed subgroup analysis and meta-regression analysis to explore the sources 
of heterogeneity. The results of subgroup analysis suggested that sample size (fewer than 100 or more than 100) altered the significance of prognostic role of CXCL5 in OS (HR 1.60, 95\% CI 0.81-3.17 vs HR 1.69, 95\% CI 1.37-2.08). This indicated that difference in sample size might be the source of heterogeneity. However, metaregression analysis failed to identify the source of the significant heterogeneity in above covariates. In addition, by combining HRs from Cox multivariate analysis, we found that CXCL5 was an independent prognostic factor of OS in cancer patients.

Furthermore, we evaluated the prognostic value of CXCL5 in certain types of cancer. We found that high CXCL5 expression was associated with reduced OS in intrahepatic cholangiocarcinoma and hepatocellular carcinoma, which was consistent with previous studies. However, there was no significant association between expression level of CXCL5 with the OS of lung cancer and colorectal cancer. For lung cancer, results from Oksana et al. were contrary to others greatly [32]. The reason might be that they only evaluated the prognostic value of CXCL5 in early stage non-small cell lung cancer (stages I and II) [32]. Similarly, for colorectal cancer, the results from Speetjens et al. also conflicted with others because they did not include stage IV patients [35, 36]. Therefore, we may speculate that CXCL5 might have different prognostic roles in different tumor stage and larger-scale, multicenter studies including all stage patients are needed to verify our hypothesis.

DFS, PFS and RFS are all important parameters reflecting the progression of tumor. Our results demonstrated that higher expression level of CXCL5 was significantly associated with shorter PFS and RFS in cancer patients. However, high or low expression of CXCL5 made no difference in predicting the DFS of cancer patients. In addition, because only three studies respectively were included to evaluate the association between CXCL5 expression levels with DFS, PFS and RFS, more studies are necessary to explore the relationship between CXCL5 with tumor progression.

Mechanisms underlying the regulatory role of CXCL5 in tumorigenesis and tumor progression have been extensively investigated. CXCL5 could activate multiple signaling pathways to promote the progression of cancer. Dai et al. found that overexpression of CXCL5 markedly upregulated the activity of the JNK, ERK and p38 MAPK signaling pathways, which may contribute to the promoting effects of CXCL5 on the proliferation and migration of glioma cells [18]. In bladder cancer, CXCL5 was found to be significantly upregulated and the CXCL5/CXCR2 axis could promote the migration and invasion of bladder cancer cells by activating the PI3K/AKT-induced upregulation of MMP2/MMP9 [19, 40]. The CXCR2/CXCL5 axis was also found to enhance epithelial-mesenchymal transition of hepatocellular carcinoma cells through the activation of the PI3K/AKT/GSK-3 $\beta /$ Snail signaling [44]. Furthermore, Hsu et al. demonstrated that progression of breast cancer induced by TAOB-derived CXCL5 was associated with increased Raf/MEK/ERK activation and mitogen- and stress-activated protein kinase 1 (MSK1) and Elk-1 phosphorylation, as well as Snail upregulation [44]. In addition, CXCL5 was shown to have potent effects on neutrophil recruitment in cancer $[45,46]$. Meanwhile, neutrophils could potentiate cancer cell migration, invasion and dissemination by secreting immunoreactive molecules such as hepatocyte growth factor, oncostatin M, b2-integrins or neutrophil elastase, which might be another mechanism for CXCL5 promoting cancer progression [27, 47, 48]. What's more, it has been reported that stem cells could produce CXCL5, and Zhao et al. demonstrated that CXCL5 secreted by adipose tissue-derived stem cells could promote breast tumor cell proliferation [49]. Thus, we could speculate that CXCL5 might be the indicator of the presence of putative cancer stem cells, which have been shown to be associated with the metastasis and poor prognosis of cancer patients [50,51].

However, the current meta-analysis had some limitations. First, the cut-off value of high and low CXCL5 expression was different among studies, which might lead to the bias of the results. Second, some HRs could not be directly obtained from the publications. Thus, calculating them through survival curves might not be precise enough. Third, differences of paper quality and sample size across the studies might cause bias in the meta-analysis, although meta-regression did not show the paper quality or sample size as the resource of heterogeneity. Therefore, larger-scale, multicenter, and high-quality studies are desperately necessary to confirm our findings.

\section{Conclusions}

In conclusion, our study revealed that elevated expression level of CXCL5 might be an adverse prognostic marker for OS, PFS and RFS in cancer patients. However, no significant association was found between CXCL5 expression level with DFS in the current meta-analysis. In a word, this is the first meta-analysis to evaluate the relationship between expression levels of CXCL5 with prognosis of cancer patients. In the future, more relevant studies are warranted to investigate the role of CXCL5 in human cancer.

\section{Abbreviations}

$\mathrm{Cl}$ : confidence intervals; HR: hazard ratios; OS: overall survival; DFS: diseasefree survival; PFS: progression-free survival; RFS: recurrence-free survival; IHC: immunohistochemistry; RT-PCR: real time polymerase chain reaction; ELISA: enzyme-linked immunosorbent assay. 


\section{Authors' contributions}

$\mathrm{BH}, \mathrm{HF}$ and $\mathrm{XL}$ collected, extracted and analyzed the data, wrote the paper; $\mathrm{XL}$ and SC performed quality assessment and analyzed the data. ZS conceived and designed this study. All authors read and approved the final manuscript.

\section{Author details}

${ }^{1}$ Department of Orthopaedics, Union Hospital, Tongji Medical College, Huazhong University of Science and Technology, Wuhan 430022, China. ${ }^{2}$ Division of Gastroenterology, Union Hospital, Tongji Medical College, Huazhong University of Science and Technology, Wuhan 430022, China. ${ }^{3}$ Department of Orthopaedic Surgery, The First Affiliated Hospital of Zhengzhou University, Zhengzhou, China.

\section{Acknowledgements}

We would like to thank the researchers and study participants for their contributions.

\section{Competing interests}

The authors declare that they have no competing interests.

\section{Availability of data and materials}

The datasets during and/or analysed during the current study available from the corresponding author on reasonable request.

\section{Consent for publication}

Not applicable.

\section{Ethics approval and consent to participate}

Not applicable.

\section{Funding}

This study was supported by Grants 2016 YFC1 100100 from The National Key Research and Development Program of China, Grants 91649204 from Major Research Plan of National Natural Science Foundation of China.

\section{Publisher's Note}

Springer Nature remains neutral with regard to jurisdictional claims in published maps and institutional affiliations.

Received: 19 March 2018 Accepted: 21 April 2018

Published online: 02 May 2018

\section{References}

1. Jemal A, Bray F, Center MM, Ferlay J, Ward E, Forman D. Global cancer statistics. CA Cancer J Clin. 2011;61(2):69-90.

2. Siegel RL, Miller KD, Jemal A. Cancer statistics, 2018. CA Cancer J Clin. 2018;68(1):7-30.

3. Shi D, Wu F, Gao F, Qing X, Shao Z. Prognostic value of long non-coding RNA CCAT1 expression in patients with cancer: a meta-analysis. PLoS ONE. 2017;12(6):e0179346.

4. Ehling J, Tacke F. Role of chemokine pathways in hepatobiliary cancer. Cancer Lett. 2016;379(2):173-83

5. Bieche I, Chavey C, Andrieu C, Busson M, Vacher S, Le Corre L, Guinebretiere J-M, Burlinchon S, Lidereau R, Lazennec G. CXC chemokines located in the $4 q 21$ region are up-regulated in breast cancer. Endocr Relat Cancer. 2007;14(4):1039-52.

6. Balkwill F. Cancer and the chemokine network. Nat Rev Cancer 2004;4(7):540-50

7. Allen SJ, Crown SE, Handel TM. Chemokine: receptor structure, interactions, and antagonism. Annu Rev Immunol. 2007;25:787-820.

8. Mellado M, Rodriguez-Frade JM, Manes S, Martinez AC. Chemokine signaling and functional responses: the role of receptor dimerization and TK pathway activation. Annu Rev Immunol. 2001;19:397-421.

9. Sallusto F, Mackay CR, Lanzavecchia A. The role of chemokine receptors in primary, effector, and memory immune responses. Annu Rev Immunol. 2000;18:593-620.

10. Lee SJ, Kim JE, Kim ST, Lee J, Park SH, Park JO, Kang WK, Park YS, Lim HY The correlation between serum chemokines and clinical outcome in patients with advanced biliary tract cancer. Transl Oncol. 2018;11(2):353-7.

11. Mantovani A, Savino B, Locati M, Zammataro L, Allavena P, Bonecchi R. The chemokine system in cancer biology and therapy. Cytokine Growth Factor Rev. 2010;21(1):27-39.

12. Allavena P, Germano G, Marchesi F, Mantovani A. Chemokines in cancer related inflammation. Exp Cell Res. 2011;317(5):664-73.

13. Mantovani A. Cancer: inflaming metastasis. Nature. 2009;457(7225):36-7.

14. Lazennec $\mathrm{G}$, Richmond A. Chemokines and chemokine receptors: new insights into cancer-related inflammation. Trends Mol Med. 2010;16(3):133-44.

15. Samarendra H, Jones K, Petrinic T, Silva MA, Reddy S, Soonawalla Z, Gordon-Weeks A. A meta-analysis of CXCL12 expression for cancer prognosis. Br J Cancer. 2017:117(1):124-35.

16. Ghoneim HM, Maher S, Abdel-Aty A, Saad A, Kazem A, Demian SR. Tumorderived CCL-2 and CXCL-8 as possible prognostic markers of breast cancer: correlation with estrogen and progestrone receptor phenotyping. Egypt J Immunol. 2009;16(2):37-48.

17. Li L, Xu L, Yan J, Zhen ZJ, Ji Y, Liu CQ, Lau WY, Zheng L, Xu J. CXCR2-CXCL1 axis is correlated with neutrophil infiltration and predicts a poor prognosis in hepatocellular carcinoma. J Exp Clin Cancer Res CR. 2015;34:129.

18. Dai Z, Wu J, Chen F, Cheng Q, Zhang M, Wang Y, Guo Y, Song T. CXCL5 promotes the proliferation and migration of glioma cells in autocrineand paracrine-dependent manners. Oncol Rep. 2016;36(6):3303-10.

19. Zhu X, Qiao Y, Liu W, Wang W, Shen H, Lu Y, Hao G, Zheng J, Tian Y. CXCL5 is a potential diagnostic and prognostic marker for bladder cancer patients. Tumour Biol. 2016:37(4):4569-77.

20. Park JY, Park KH, Bang S, Kim MH, Lee JE, Gang J, Koh SS, Song SY. CXCL5 overexpression is associated with late stage gastric cancer. J Cancer Res Clin Oncol. 2007;133(11):835-40.

21. Kuo PL, Chen YH, Chen TC, Shen KH, Hsu YL CXCL5/ENA78 increased cell migration and epithelial-to-mesenchymal transition of hormoneindependent prostate cancer by early growth response-1/snail signaling pathway. J Cell Physiol. 2011;226(5):1224-31.

22. Wong YF, Cheung TH, Lo KWK, Yim SF, Siu NSS, Chan SCS, Ho TWF Wong KWY, Yu MY, Wang VW, et al. Identification of molecular markers and signaling pathway in endometrial cancer in Hong Kong Chinese women by genome-wide gene expression profiling. Oncogene. 2007;26(13):1971-82

23. Miyazaki H, Patel V, Wang H, Edmunds RK, Gutkind JS, Yeudall WA. Downregulation of CXCL5 inhibits squamous carcinogenesis. Cancer Res. 2006:66(8):4279-84

24. Zhou S-L, Dai Z, Zhou Z-J, Wang X-Y, Yang G-H, Wang Z, Huang X-W, Fan J, Zhou J. Overexpression of CXCL5 mediates neutrophil infiltration and indicates poor prognosis for hepatocellular carcinoma. Hepatology. 2012;56(6):2242-54

25. Li A, King J, Moro A, Sugi MD, Dawson DW, Kaplan J, Li G, Lu X, Strieter RM Burdick M, et al. Overexpression of CXCL5 is associated with poor survival in patients with pancreatic cancer. Am J Pathol. 2011:178(3):1340-9.

26. Yu S, Liu Q, Bai X, Zheng X, Wu K. The clinical significance of CXCL5 in non-small cell lung cancer. OncoTargets Ther. 2017:10:5561-73.

27. Zhou S-L, Dai Z, Zhou Z-J, Chen Q, Wang Z, Xiao Y-S, Hu Z-Q, Huang X-Y, Yang G-H, Shi Y-H, et al. CXCL5 contributes to tumor metastasis and recurrence of intrahepatic cholangiocarcinoma by recruiting infiltrative intratumoral neutrophils. Carcinogenesis. 2014:35(3):597-605.

28. Altman DG, MCShane LM, Sauerbrei W, Taube SE. Reporting recommendations for tumor marker prognostic studies (REMARK): explanation and elaboration. PLoS Med. 2012:9(5):e1001216.

29. McShane LM, Altman DG, Sauerbrei W, Taube SE, Gion M, Clark GM Reporting recommendations for tumor marker prognostic studies (REMARK). J Natl Cancer Inst. 2005;97(16):1180-4.

30. Parmar MK, Torri $V$, Stewart L. Extracting summary statistics to perform meta-analyses of the published literature for survival endpoints. Stat Med. 1998:17(24):2815-34

31. Okabe H, Beppu T, Ueda M, Hayashi H, Ishiko T, Masuda T, Otao R, Horlad H, Mima K, Miyake K, et al. Identification of CXCL5/ENA-78 as a factor involved in the interaction between cholangiocarcinoma cells and cancer-associated fibroblasts. Int J Cancer. 2012;131(10):2234-41.

32. Kowalczuk O, Burzykowski T, Niklinska WE, Kozlowski M, Chyczewski L, Niklinski J. CXCL5 as a potential novel prognostic factor in early stage 
non-small cell lung cancer: results of a study of expression levels of 23 genes. Tumour Biol. 2014;35(5):4619-28.

33. Han N, Yuan X, Wu H, Xu H, Chu Q, Guo M, Yu S, Chen Y, Wu K. DACH1 inhibits lung adenocarcinoma invasion and tumor growth by repressing CXCL5 signaling. Oncotarget. 2015;6(8):5877-88.

34. Kawamura M, Toiyama Y, Tanaka K, Saigusa S, Okugawa Y, Hiro J, Uchida K, Mohri Y, Inoue Y, Kusunoki M. CXCL5, a promoter of cell proliferation, migration and invasion, is a novel serum prognostic marker in patients with colorectal cancer. Eur J Cancer. 2012:48(14):2244-51.

35. Speetjens FM, Kuppen PJK, Sandel MH, Menon AG, Burg D, van Velde CJH, Tollenaar RAEM, de Bont HJGM, Nagelkerke JF. Disrupted expression of CXCL5 in colorectal cancer is associated with rapid tumor formation in rats and poor prognosis in patients. Clin Cancer Res. 2008;14(8):2276-84.

36. Zhao J, Ou B, Han D, Wang P, Zong Y, Zhu C, Liu D, Zheng M, Sun J, Feng $H$, et al. Tumor-derived CXCL5 promotes human colorectal cancer metastasis through activation of the ERK/Elk-1/Snail and AKT/GSK3 $/ / \beta$-catenin pathways. Mol Cancer. 2017;16(1):70.

37. Zhang H, Xia W, Lu X, Sun R, Wang L, Zheng L, Ye Y, Bao Y, Xiang Y, Guo X. A novel statistical prognostic score model that includes serum CXCL5 levels and clinical classification predicts risk of disease progression and survival of nasopharyngeal carcinoma patients. PLoS ONE. 2013;8(2):e57830.

38. Strieter RM, Burdick MD, Gomperts BN, Belperio JA, Keane MP. CXC chemokines in angiogenesis. Cytokine Growth Factor Rev. 2005;16(6):593-609.

39. Persson T, Monsef N, Andersson P, Bjartell A, Malm J, Calafat J, Egesten A. Expression of the neutrophil-activating CXC chemokine ENA-78/CXCL5 by human eosinophils. Clin Exp Allergy. 2003:33(4):531-7.

40. Gao Y, Guan Z, Chen J, Xie H, Yang Z, Fan J, Wang X, Li L. CXCL5/CXCR2 axis promotes bladder cancer cell migration and invasion by activating PI3K/AKT-induced upregulation of MMP2/MMP9. Int J Oncol. 2015:47(2):690-700

41. Wente MN, Keane MP, Burdick MD, Friess H, Buchler MW, Ceyhan GO, Reber HA, Strieter RM, Hines OJ. Blockade of the chemokine receptor CXCR2 inhibits pancreatic cancer cell-induced angiogenesis. Cancer Lett. 2006;241(2):221-7.

42. Takahashi H, Numasaki M, Lotze MT, Sasaki H. Interleukin-17 enhances bFGF-, HGF- and VEGF-induced growth of vascular endothelial cells. Immunol Lett. 2005:98(2):189-93.
43. Arenberg DA, Keane MP, DiGiovine B, Kunkel SL, Morris SB, Xue YY, Burdick MD, Glass MC, lannettoni MD, Strieter RM. Epithelial-neutrophil activating peptide (ENA-78) is an important angiogenic factor in non-small cell lung cancer. J Clin Investig. 1998;102(3):465-72.

44. Zhou SL, Zhou ZJ, Hu ZQ, Li X, Huang XW, Wang Z, Fan J, Dai Z, Zhou J. CXCR2/CXCL5 axis contributes to epithelial-mesenchymal transition of HCC cells through activating PI3 K/Akt/GSK-3beta/Snail signaling. Cancer Lett. 2015;358(2):124-35.

45. Zhou SL, Dai Z, Zhou ZJ, Wang XY, Yang GH, Wang Z, Huang XW, Fan J, Zhou J. Overexpression of CXCL5 mediates neutrophil infiltration and indicates poor prognosis for hepatocellular carcinoma. Hepatology. 2012;56(6):2242-54

46. Zhou SL, Dai Z, Zhou ZJ, Chen Q, Wang Z, Xiao YS, Hu ZQ, Huang XY, Yang $\mathrm{GH}$, Shi YH, et al. CXCL5 contributes to tumor metastasis and recurrence of intrahepatic cholangiocarcinoma by recruiting infiltrative intratumoral neutrophils. Carcinogenesis. 2014:35(3):597-605.

47. Imai Y, Kubota Y, Yamamoto S, Tsuji K, Shimatani M, Shibatani N, Takamido S, Matsushita M, Okazaki K. Neutrophils enhance invasion activity of human cholangiocellular carcinoma and hepatocellular carcinoma cells: an in vitro study. J Gastroenterol Hepatol. 2005;20(2):287-93.

48. Brandau S, Dumitru CA, Lang S. Protumor and antitumor functions of neutrophil granulocytes. Semin Immunopathol. 2013;35(2):163-76.

49. Zhao Y, Zhang X, Zhao H, Wang J, Zhang Q. CXCL5 secreted from adipose tissue-derived stem cells promotes cancer cell proliferation. Oncol Lett. 2018;15(2):1403-10.

50. Smith BA, Sokolov A, Uzunangelov V, Baertsch R, Newton Y, Graim K, Mathis C, Cheng D, Stuart JM, Witte ON. A basal stem cell signature identifies aggressive prostate cancer phenotypes. Proc Natl Acad Sci USA. 2015;112(47):E6544-52.

51. Finicelli M, Benedetti G, Squillaro T, Pistilli B, Marcellusi A, Mariani P, Santinelli A, Latini L, Galderisi U, Giordano A. Expression of stemness genes in primary breast cancer tissues: the role of SOX2 as a prognostic marker for detection of early recurrence. Oncotarget. 2014:5(20):9678-88.
Ready to submit your research? Choose BMC and benefit from:

- fast, convenient online submission

- thorough peer review by experienced researchers in your field

- rapid publication on acceptance

- support for research data, including large and complex data types

- gold Open Access which fosters wider collaboration and increased citations

- maximum visibility for your research: over $100 \mathrm{M}$ website views per year

At $\mathrm{BMC}$, research is always in progress.

Learn more biomedcentral.com/submissions 